\title{
Auriculoterapia no manejo da ansiedade em estudantes universitários: um estudo randomizado
}

\author{
Auriculotherapy in anxiety management in university students: a randomized study
}

Auriculoterapia en el manejo de la ansiedad en estudiantes universitarios: un estudio aleatorizado

Ricardo Andre Medeiros Negreiros ${ }^{1 *}$, Vinícius Matos Formiga1, Jose Vitor Alecio Rodrigues ${ }^{1}$, Ana Quezia Bezerra de Holanda Sousa ${ }^{1}$, Ian Félix Martins Costa ${ }^{1}$, Climerio Avelino de Figueiredo ${ }^{1}$, Maria do Socorro Trindade Morais ${ }^{1}$, Jacicarlos Lima de Alencar ${ }^{1}$, Liane Franco Barros Mangueira ${ }^{1}$.

\section{RESUMO}

Objetivo: Avaliar a efetividade da acupuntura auricular na redução dos níveis de ansiedade em estudantes universitários, quando comparada com o placebo. Métodos: Foram randomizados 40 voluntários em dois grupos: Auriculoterapia com sementes seguindo o protocolo para Ansiedade (G1) e Placebo (G2). Os grupos foram acompanhados durante seis semanas, com aplicações semanais da auriculoacupuntura, e responderam o Inventário de Ansiedade Traço (IDATE-T) ao iniciarem a primeira sessão e o Inventário de Ansiedade Estado (IDATE-E) na primeira e última sessões. Resultados: Os participantes do G1 obtiveram escore médio do IDATE-T de 46,45 pontos, enquanto o IDATE-E inicial e o final foram, respectivamente, 45,76 e 37,05. O G2 obteve os valores de 49,3 para o IDATE-T, 44,3 e 43,3 para o IDATE-E inicial e final. O teste dos pontos com sinais de Wilcoxon demonstrou significância estatística para a redução dos escores de ansiedade no G1, o que não foi observado no G2. Conclusão: O estudo quantificou os escores de ansiedade na população estudantil universitária e a possibilidade de reduzi-los a partir da proposta terapêutica da auriculoacupuntura. Foi observada redução nos escores de ansiedade nos participantes dos grupos estudados, entretanto, quando comparados os resultados, apenas houve significância estatística no grupo com protocolo para ansiedade.

Palavras-chave: Auriculoterapia, Ansiedade, Estresse psicológico, Saúde do estudante.

\begin{abstract}
Objective: Evaluate the effectiveness of auricular acupuncture in reducing anxiety levels in college students when compared to placebo. Methods: Forty volunteers were randomized into two groups: Auriculotherapy with seeds following the protocol for Anxiety (G1) and Placebo (G2). The groups were followed for six weeks, with weekly applications of auriculoacupuncture, and answered the Trait Anxiety Inventory (IDATE-T) when starting the first session and the State Anxiety Inventory (IDATE-E) in the first and last sessions. Results: G1 participants obtained a mean IDATE-T score of 46.45 points, while the initial and final IDATE-E were 45.76 and 37.05 , respectively. G2 obtained the values of 49.3 for the IDATE-T, 44.3 and 43.3 for the initial and final IDATE-E. The Wilcoxon sign-point test demonstrated statistical significance for the reduction of anxiety scores in G1, which was not observed in G2. Conclusion: The study quantified the anxiety scores in the university student population and the possibility of reducing them from the auriculoacupuncture therapeutic proposal. Reduction in anxiety scores was observed in participants of the groups studied, however, when results were compared, there was statistical significance only in the group with protocol for anxiety.
\end{abstract}

Key words: Auriculotherapy, Anxiety, Stress psychological, Student health.

1 Universidade Federal da Paraíba, João Pessoa - PB. *E-mail: ricardoandrenegreiros@gmail.com 


\section{RESUMEN}

Objetivo: Evaluar la eficacia de la acupuntura auricular para reducir los niveles de ansiedad en estudiantes universitarios en comparación con el placebo. Métodos: Cuarenta voluntarios fueron distribuidos aleatoriamente en dos grupos: Auriculoterapia con semillas siguiendo el protocolo para la Ansiedad (G1) y Placebo (G2). Los grupos fueron seguidos durante seis semanas, con aplicaciones semanales de auriculoacupuntura, y respondieron al Inventario de Ansiedad de Rasgo (IDATE-T) al iniciar la primera sesión y al Inventario de Ansiedad de Estado (IDATE-E) en la primera y última sesión. Resultados: Los participantes del G1 obtuvieron una puntuación media de IDATE-T de 46,45 puntos, mientras que las puntuaciones iniciales y finales de IDATE-E fueron de 45,76 y 37,05, respectivamente. G2 obtuvo los valores de 49,3 para el IDATET, 44,3 y 43,3 para el IDATE-E inicial y final. La prueba del punto de signo de Wilcoxon mostró significación estadística para la reducción de las puntuaciones de ansiedad en G1, que no se observó en G2. Conclusión: El estudio cuantificó las puntuaciones de ansiedad en la población estudiantil universitaria y la posibilidad de reducirlas a partir de la propuesta terapéutica de la auriculoacupuntura. Se observó una reducción de las puntuaciones de ansiedad en los participantes de los grupos estudiados, sin embargo, cuando se compararon los resultados, sólo hubo significación estadística en el grupo con protocolo para la ansiedad.

Palabras clave: Auriculoterapia, Ansiedad, Estrés psicológico, Salud del estudiante.

\section{INTRODUÇÃO}

O estresse é definido como um estado no qual o equilíbrio dinâmico do corpo (homeostase) é ameaçado por eventos ou fatores adversos internos ou externos, conhecidos como estressores, repercutindo no organismo com diversas respostas adaptativas (GODOY LD, et al., 2018). Dentre elas, há efeitos no sistema nervoso e límbico, podendo causar mudanças em diferentes partes do corpo. A intensidade e tempo de duração da exposição dos indivíduos a essas situações de estresse podem gerar sequelas significativas, com diminuição da imunidade e prejuízo da qualidade de vida (YARIBEYGI H, et al., 2017).

Em estimativas da Organização Mundial da Saúde (WHO) (2017), foi demonstrado que grande parcela da população brasileira, 18,6 milhões de habitantes $(9,3 \%)$ sofre de ansiedade e 11,5 milhões $(5,8 \%)$ apresentam desordens depressivas, maior taxa populacional em comparativo com os outros países do mundo.

A ansiedade é a antecipação de perigo que gera o sentimento de desconforto, tensão, apreensão e medo (CROCQ MA, 2015). Apesar de ser considerada uma resposta fisiológica em situações de estresse físico e psicológico, estimulando a reação do indivíduo, a ansiedade também tem um potencial nocivo, visto que tende à cronicidade, afetando diretamente o cotidiano e aumentando o risco de desenvolvimento de doenças secundárias (BRASIL, 2011; ERRINGTON-EVANS N, 2012). Os transtornos de ansiedade incluem uma série de afecções, tais como Transtorno de Pânico, Transtorno Obsessivo-Compulsivo, Transtorno de Estresse Pós-Traumático, Fobia Social, Fobia específica, Transtorno de Ansiedade Generalizada e ansiedade secundária à condição clínica subjacente (KUREBAYASHI LFS, et al., 2017).

Os sintomas clássicos do Transtorno Depressivo são a anedonia, o humor depressivo e a ansiedade, afetando diretamente a qualidade de vida do indivíduo. É considerada uma doença relacionada ao estresse, especialmente aquele causado por eventos negativos na vida, o que contribui para a manifestação e neuroprogressão da doença (PEREIRA C, et al., 2019). Dentre outros sintomas característicos, encontramse alterações psicossomáticas, além da sensação de tristeza, desânimo, tendência ao isolamento, insônia, redução do apetite e autoestima (WU C, et al., 2018).

A prevalência dessas condições tem relação com as atividades laborais vivenciadas por profissionais de diversas áreas. Os profissionais de saúde, especialmente, estão diretamente expostos às situações de estresse derivadas de suas atividades, o que aumenta o risco de desenvolvimento de males como a Síndrome de Burnout, Transtorno de Estresse Pós-Traumático secundário, entre outros. O estresse provocado por essas e outras disfunções está associado a uma maior vulnerabilidade ao uso abusivo de drogas lícitas e ilícitas, bem como ao cansaço, à insônia, à exaustão e às alterações emocionais, como a ansiedade e a 
depressão (BUCHANAN TM, et al., 2018). Sugere-se ainda uma provável correlação entre a síndrome de Burnout e o desenvolvimento de transtornos de ansiedade, especialmente quando em um ambiente laboral desfavorável (OLSHAN-PERLMUTTER M, et al., 2019).

Nesse cenário, os estudantes universitários se revelam como um grupo de risco em potencial, pois são submetidos a situações de desgaste físico e mental, o que pode reduzir o desempenho acadêmico ou, ainda, contribuir para as elevadas taxas de transtornos mentais observadas nestes grupos (KEYES CLM, et al., 2012; ROTENSTEIN LS, et al., 2016). Os fatores desencadeantes do sofrimento psíquico nessa população estão relacionados a percepção e características do curso, aos desempenhos acadêmico e educacional (GRANER KM e CERQUEIRA ATDAR, 2019).

As práticas integrativas e complementares se apresentam como modalidades de cuidado e são frequentemente requisitadas por pacientes sob dificuldades emocionais, físicas e psíquicas (HALLER $\mathrm{H}$, et al., 2019). No Brasil, sua realização foi institucionalizada pela Política Nacional de Práticas Integrativas e Complementares em Saúde (PNPIC) implementada no Sistema Único de Saúde (SUS) em 2006, e são definidas como recursos terapêuticos que tomam por base os saberes tradicionais para desenvolver atividades que auxiliam na prevenção de doenças e no alívio dos sintomas, sem substituição do tratamento convencional (BRASIL, 2015).

A medicina tradicional chinesa é parte constituinte das Práticas Integrativas e Complementares, sendo a acupuntura uma importante componente destas práticas milenares. Essa já possui embasamento científico que demonstra efeitos positivos no manejo de diferentes modalidades de dor, além de efeitos sobre a ansiedade e sintomas depressivos (AMORIM D, et al., 2018; SMITH CA, et al., 2018; VICKERS AJ, et al., 2012).

A acupuntura auricular, adjuvante da acupuntura sistêmica, é utilizada na China desde a dinastia Han, fundamentada nas conexões entre a orelha externa e as diversas partes do corpo, sendo possível realizar um diagnóstico a partir da inspeção auricular e tratá-lo através da estimulação de pontos localizados no pavilhão auricular (MOURA CDC, et al., 2019a; WIRZ-RIDOLFI A, 2019).

Na contemporaneidade, reconhece-se que a auriculoterapia possui dois ramos: 0 da Medicina Tradicional Chinesa (MTC) e o francês. A MTC é composta pelo diagnóstico da doença a partir da inspeção da orelha, visto que as mudanças psicológicas ou patológicas no corpo podem ecoar no pavilhão auricular, relacionandoo aos órgãos internos por meio de canais, criando assim zonas correspondentes a serem estimuladas. Já o método francês advém da pesquisa sistêmica do neurologista Paul Nogier, que, a partir de suas observações, concluiu que a correspondência reflexa na orelha era análoga a um mapa em formato de feto invertido, representando o local dos pontos de estímulos dos órgãos. Nogier também descreveu que o estímulo auricular reflexo a zonas patologicamente alteradas desencadeava uma mudança no pulso cardíaco do paciente, o que ficou conhecido como "Sinal Autônomo Vascular", marcando o início do que viria a se chamar a auriculomedicina ou auriculoterapia moderna (LAN et al., 2015; WIRZ-RIDOLFI A, 2019).

A auriculoacupuntura, após as políticas públicas que a implementaram no SUS, tem sido difundida como uma terapia complementar com boa aceitação pelas comunidades assistidas. Para o estímulo, várias técnicas são possíveis, dentre elas, a utilização de agulhas, sementes de Vaccaria, sondas e laser (RABISCHONG $P$ e TERRAL C, 2014). É reconhecido que o mecanismo de ação da auriculoterapia ainda necessita ser esclarecido, porém já há estudos que fortalecem a ideia de que esse tratamento possui efeitos positivos para dor crônica, insônia e cessação do tabagismo. Todavia, a baixa qualidade e variedade dos desenhos metodológicos dificultam a comprovação dos resultados descritos (VIEIRA A, et al., 2018).

A facilidade na aplicação e o baixo risco dos efeitos colaterais e adversos são aspectos favoráveis da acupuntura auricular, que pode ser de grande valia para o tratamento complementar de diversas patologias. O objetivo do estudo foi avaliar a efetividade da terapia auricular para a redução de ansiedade em estudantes de uma universidade pública que buscaram atendimento no Centro de Referência em Atenção à Saúde (CRAS) existente no Campus Universitário, além de apresentar aos estudantes e profissionais de saúde a perspectiva de utilização desta prática no manejo do sofrimento psíquico. 


\section{MÉTODOS}

Trata-se de um ensaio clínico randomizado realizado no Centro de Referência em Atenção à Saúde (CRAS), no período de agosto de 2019 a março de 2020. Os critérios de inclusão adotados para a pesquisa foram: ser estudante com vínculo ativo na universidade onde foi realizado o estudo, maiores de 18 anos, que buscaram atendimento no CRAS. Como critérios de exclusão: gestantes, nutrizes, indivíduos que estivessem em uso de psicotrópicos ou que não possuíssem capacidade de entender e responder os questionários, recusarem-se a receber a intervenção ou a assinar o Termo de Consentimento Livre e Esclarecido. A pesquisa foi aprovada pelo Comitê de Ética em Pesquisa (CEP) sob o número do parecer: 3.292.550. Os voluntários foram divididos de forma aleatória, respeitando os critérios de inclusão e de exclusão.

Ao início da pesquisa, utilizou-se o programa Research Randomizes Quick Tutorial para alocar os participantes entre os grupos: G1 - Auriculoterapia com sementes seguindo o protocolo para ansiedade; G2 - Auriculoterapia com sementes seguindo o protocolo Placebo. O protocolo para ansiedade consiste nos pontos: Shen Men, neurastenia e ansiedade. Já o protocolo Placebo, representando pontos não correspondentes aos sintomas ou tratamento de ansiedade, é formado pelos pontos: dedos do pé, maxilar superior e ouvido externo.

O período de intervenção para cada voluntário foi de seis semanas, com cinco sessões para aplicação da acupuntura auricular e uma sexta para aplicação final dos questionários. Utilizaram-se os Inventários de Ansiedade Traço-Estado (IDATE), instrumentos de avaliação dos níveis de ansiedade criados por Spielbergert, Gorsuch e Lushene em 1970. É um dos recursos mais utilizados para quantificar a ansiedade por ser composto por duas partes: estado (IDATE-E), referente a situações transitórias e momentâneas dos últimos dias, e traço (IDATE-T), correspondente a aspectos e tendências estáveis de como o indivíduo lida com maior ou menor ansiedade ao longo de semanas. O IDATE considera a pontuação mínima de 20 pontos e máxima de 80 pontos, considerando-se o escore de 20 a 40 como o indivíduo levemente ansioso, 41 a 60 como ansioso moderado, e 61 a 80 como portador de ansiedade grave, podendo estar relacionado a algum transtorno de ansiedade ou depressão, sendo cabível encaminhamento para auxílio psiquiátrico.

Durante a primeira sessão, foi realizada a aplicação dos IDATE, nas suas modalidades IDATE-T e IDATE$\mathrm{E}$, além do tratamento com auriculoacupuntura unilateral. Seguiam-se os encontros semanais para remover os pontos do pavilhão auricular e realizar nova aplicação do protocolo definido previamente, até a última semana, sendo retirados os pontos e preenchido o IDATE-E final pelos voluntários participantes da pesquisa.

No começo da pesquisa, foram considerados 40 estudantes, distribuídos igualmente entre os dois grupos. No decorrer do estudo, 3 voluntários do G1 abandonaram a pesquisa, representando cerca de $15 \%$ da amostra do grupo, enquanto 10 do G2 também desistiram (50\%). Ocorreu uma perda total de $13(32,5 \%)$ dos indivíduos.

Para a análise dos dados, foi utilizado o software Statistical Package for the Social Sciences (SPSS), versão 24. A análise descritiva utilizou medidas de frequências relativas e absolutas, comparando os resultados encontrados entre os grupos, além de traçar medianas e tendências entre os questionários ao longo da pesquisa. Para avaliar a significância estatística da diferença entre o IDATE-E inicial e final, utilizouse o Teste dos Postos com sinal de Wilcoxon, determinando a significância dos grupos com protocolo para ansiedade e placebo. Foi realizado ainda o teste de Mann-Whitney, para buscar diferenças significativas entre os grupos avaliados.

A natureza randômica dos grupos e a escolha de protocolos predefinidos dos pontos a serem aplicados durante as sessões de auriculoacupuntura, em vez do tratamento auricular individualizado e focado nas queixas específicas trazidas pelos pacientes, proporcionaram a minimização de possíveis vieses. Acredita-se que os resultados díspares, entre o controle e o placebo, não tenham ocorrido por um viés de ocultação (unblinding), a maioria dos voluntários não tinha conhecimento extenso sobre técnicas de tratamento com auriculoterapia, além disso, caso ocorresse a percepção do protocolo Placebo, poderia não ter sido observado o resultado encontrado durante o período da execução da pesquisa. 


\section{RESULTADOS}

Dentre os participantes da pesquisa ( $n=40)$, foram alocados vinte no $G 1$ e vinte no $G 2$, todos responderam aos IDATE-E inicial e IDATE-T. Destes, ocorreu uma perda de três voluntários do G1 (15\%) e dez do G2 $(50 \%)$, durante as sessões. Os participantes desistentes alegaram falta de disponibilidade de tempo $(n=6)$, irritação cutânea auricular ( $n=3)$ e não forneceram explicação $(n=4)$. Ao fim da pesquisa, após as 5 aplicações, vinte e sete participantes, sendo dezessete do G1 e dez do G2, responderam ao IDATE-E final.

Na linha de base, os participantes do G1 apresentaram escore médio de ansiedade-traço de 46,65 $( \pm 11,53)$ e mediana de 46 (37-54,5); ansiedade-estado inicial, com média de 45,76 $( \pm 12,16)$ e mediana $42(36,5-56,5)$. O grupo com protocolo placebo mostrou escore médio de ansiedade-traço, com média de $49,3( \pm 7,89)$ e mediana de $48(43,5-55,5)$ e escore médio de ansiedade-estado inicial, com média de $44,3( \pm 11,86)$ e mediana de 45,5 (35,5-56) (Tabela 1 e Tabela 2).

Tabela 1 - Frequência absoluta e relativa de ansiedade do grupo G1.

\begin{tabular}{c|c|c}
\hline Ansiedade Traço & $\mathbf{N = 1 7}$ & $\%$ \\
\hline Levemente Ansioso $(20-40)$ & 6 & 35.29 \\
Ansiedade Moderada $(41-60)$ & 8 & 47.06 \\
Ansiedade Grave $(61-80)$ & 3 & 17.65 \\
\hline Ansiedade Estado Inicial & $\mathbf{N = 1 7}$ & $\%$ \\
\hline Levemente Ansioso $(20-40)$ & 8 & 47.06 \\
Ansiedade Moderada (41- 60) & 7 & 41.18 \\
Ansiedade Grave $(61-80)$ & 2 & 11.76 \\
\hline
\end{tabular}

Fonte: Negreiros RAM, et al., 2021.

Tabela 2 - Frequência absoluta e relativa de ansiedade do grupo G2.

\begin{tabular}{c|c|c}
\hline Ansiedade Traço & $\mathbf{N = 1 0}$ & $\%$ \\
\hline Levemente Ansioso (20 - 40) & 1 & 10 \\
Ansiedade Moderada (41-60) & 8 & 80 \\
Ansiedade Grave (61-80) & 1 & 10 \\
\hline Ansiedade Estado Inicial & $\mathbf{N = 1 0}$ & $\%$ \\
\hline Levemente Ansioso (20 - 40) & 4 & 40 \\
Ansiedade Moderada (41-60) & 6 & 60 \\
Ansiedade Grave (61-80) & 0 & 0 \\
\hline
\end{tabular}

Fonte: Negreiros RAM, et al., 2021.

Ao final das sessões, os participantes do grupo com protocolo para ansiedade exibiram escore ansiedade-estado final inferior, com média de $37,05( \pm 8,00)$, representando uma redução percentual de 19,03\%, e mediana de 37 (29-41), que reduziu 11,90\%. O G2 também apresentou escore ansiedade-estado inferior, com média de $43,3( \pm 10,4)$, com redução de $2,26 \%$ e mediana de $43,5(36,75-49)$, que reduziu $4,40 \%$ (Tabela 3, Gráfico 1 e Gráfico 2).

Tabela 3 - Frequência absoluta e relativa de ansiedade-estado final G1 x G2.

\begin{tabular}{c|c|c}
\hline Ansiedade Estado Final (G1) & $\mathbf{N = 1 7}$ & $\%$ \\
\hline Levemente Ansioso (20 - 40) & 12 & 70.59 \\
Ansiedade Moderada (41 - 60) & 5 & 29.41 \\
Ansiedade Grave (61 - 80) & 0 & 0 \\
\hline Ansiedade Estado Final (G2) & $\mathbf{N}=\mathbf{1 0}$ & $\%$ \\
\hline Levemente Ansioso (20 - 40) & 3 & 30 \\
Ansiedade Moderada (41 - 60) & 6 & 60 \\
Ansiedade Grave (61 - 80) & 1 & 10 \\
\hline
\end{tabular}

Fonte: Negreiros RAM, et al., 2021. 


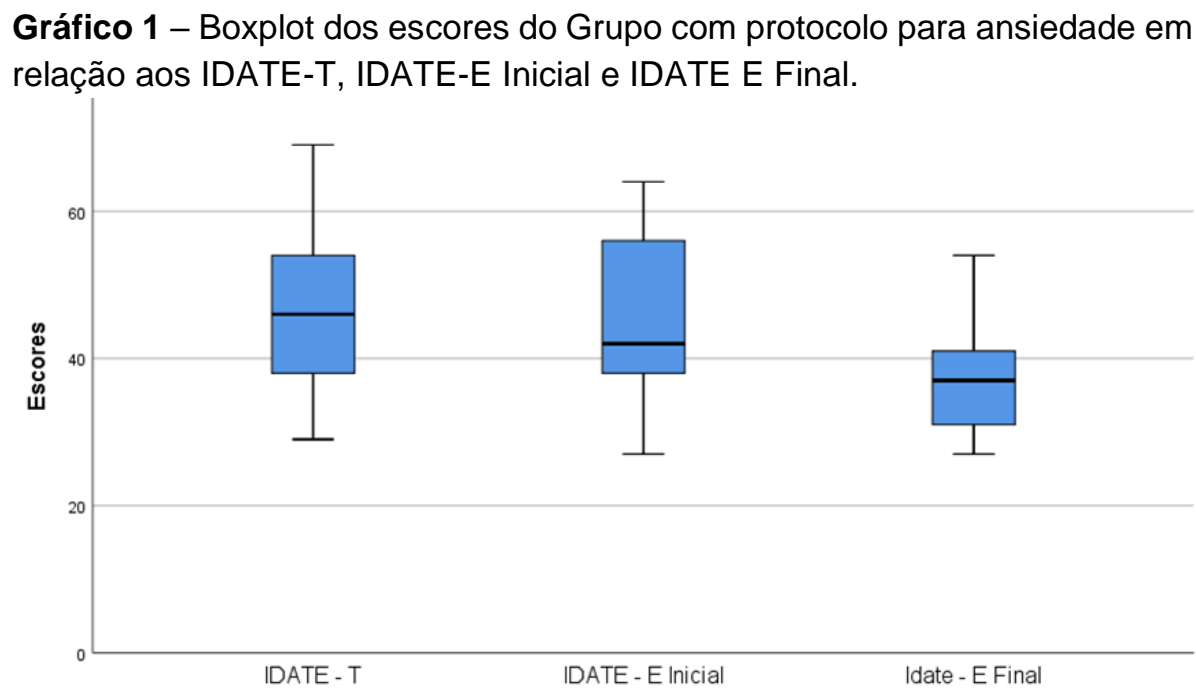

Fonte: Negreiros RAM, et al., 2021.

Gráfico 2 - Boxplot dos escores do Grupo com protocolo placebo em relação aos IDATE-T, IDATE-E Inicial e IDATE E Final.

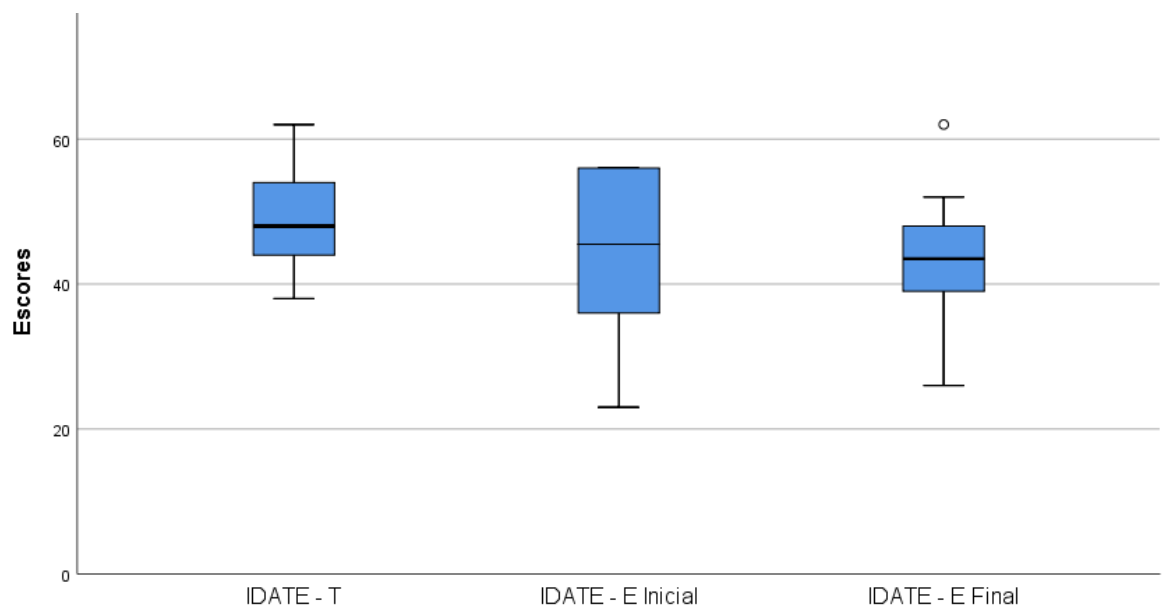

Fonte: Negreiros RAM, et al., 2021.

O teste de normalidade de D'Agostino \& Pearson reconheceu distribuição normal para os dados do IDATE$\mathrm{T}(\mathrm{K} 2=0,53 ; p=0,76)$, IDATE-E inicial $(\mathrm{K} 2=4,45 ; \mathrm{p}=0,11)$ e IDATE-E final $(\mathrm{K} 2=1,74 ; \mathrm{p}=0,42)$, no grupo com protocolo para ansiedade. O mesmo teste também reconheceu distribuição normal para os dados de IDATE$\mathrm{T}(\mathrm{K} 2=0,73 ; p=0,69)$, IDATE-E inicial $(\mathrm{K} 2=1,19 ; \mathrm{p}=0,55)$ e IDATE-E final $(\mathrm{K} 2=0,20 ; p=0,91)$, no grupo com protocolo placebo.

Na comparação entre IDATE-E final e IDATE-E inicial, para o G1 $(n=17): 12(70,6 \%)$ apresentaram postos negativos, situações em que IDATE-E final foi menor que o IDATE-E inicial (posto médio de 10,50); 5 (29,4\%) apresentaram postos positivos, situações em que IDATE-E final foi maior que o IDATE-E inicial (posto médio de 5,40); e não foram observados casos em que o IDATE-E final fosse igual ao IDATE-E inicial. Para o G2 $(n=10)$ : 5 voluntários (50\%) apresentaram postos negativos (posto médio de 10,50); 5 (50\%) apresentaram postos positivos (posto médio de 5,40), de maneira semelhante, não se observou situações em que o IDATE$E$ final fosse igual ao IDATE-E inicial.

O teste dos pontos com sinais de Wilcoxon mostrou significância estatística para redução de escores de ansiedade, ao final do tratamento, para o grupo com protocolo para ansiedade $(Z=-2,345 ; p=0,019)$. O mesmo teste não obteve significância para o grupo com protocolo placebo $(Z=-0,255 ; p=0,799)$. 
O teste de Mann-Whitney demonstrou que a realização de auriculoterapia em grupo com protocolo para ansiedade ou placebo, antes do início da terapia, não interferiu nos escores de ansiedade-traço $(U=73,5$, $\mathrm{p}=0,563)$ ou ansiedade-estado inicial $(U=77,0, p=0,687)$. Ao final das sessões, o mesmo teste também não demonstrou interferência da aplicação para ansiedade ou placebo nos escores ansiedade-estado final $(U=51,0, p=0,087)$. Apesar de não encontrada diferença estatística significante quanto ao posicionamento reflexo dos pontos auriculares, ocorreu brusca variação entre os valores da probabilidade de significância $(p)$ quando comparados os IDATE iniciais aos finais dos dois grupos.

\section{DISCUSSÃO}

O meio universitário é característico por apresentar os alunos a demandas emocionais, físicas e mentais, causando estresse e os afetando negativamente, podendo resultar em depressão, ansiedade e outras formas de adoecimento psíquico, além de sintomas como fadiga, cefaleia, dor abdominal, palpitações e falta de ar (HASTY LM, et al., 2020; QUEK TTC, et al., 2019).

Quando a ansiedade dos estudantes é alta, há a tendência de diminuição do rendimento acadêmico, devido à incidência de comportamentos de fuga. Os estudantes, que deveriam estar tendo oportunidades de aprender e crescer, acabam por ter sua qualidade de vida e aprendizado prejudicados (HASTY LM, et al., 2020). O presente estudo, desenvolvido com determinada população, mostrou-se útil para quantificar os escores de ansiedade observados na mesma e a possibilidade de reduzi-los a partir da proposta terapêutica da auriculoacupuntura.

O estudo realizado por Klausenitz C (2016) é o que mais se assemelha a esta pesquisa, executado com 44 estudantes de graduação do curso de medicina, quantificando a ansiedade destes em períodos de atividades avaliativas. Nos três grupos randomizados, o primeiro recebeu a auriculoterapia $(n=11)$, o segundo recebeu placebo $(n=12)$ e o terceiro não foi submetido a nenhuma intervenção $(n=11)$, foi feito o acompanhamento do escore de ansiedade ao longo de três momentos: antes da intervenção, após a intervenção e na manhã antes do exame. As médias de pontuação do IDATE-T que encontraram antes da intervenção foram: 44,45 e 43 respectivamente, enquanto o IDATE-E no mesmo período gerou os valores de 55, 53 e 54. Observou-se, após o momento de intervenção, pelo IDATE-E, redução de 8 pontos no escore daqueles que receberam aplicação da auriculoterapia, 1 ponto nos que fizeram uso de placebo e aumento de 3 pontos em quem não recebeu intervenção (KLAUSENITZ C, et al., 2016). Percebe-se, a partir destes dados, que ambas as formas de intervenção geram redução da ansiedade, entretanto, quando comparado ao placebo, a auriculoacupuntura possui maior eficácia. Por utilizar os mesmos parâmetros para a avaliação dos escores de ansiedade (IDATE-T e IDATE-E), a pesquisa reforça a tese defendida neste estudo.

Outras pesquisas realizadas com estudantes universitários, como a desenvolvida por CHUEH KH (2018), realça não apenas os efeitos da acupuntura auricular para a redução da ansiedade, como outros aspectos estressores que causam sofrimento. Acompanhando 36 estudantes de enfermagem ao longo de quatro semanas de intervenção, demonstrou-se efeito positivo no uso de auriculoterapia para a melhora de ansiedade, humor depressivo e qualidade do sono, com redução de $26,7 \%, 43,5 \%$ e $25 \%$, respectivamente, nos participantes da pesquisa (CHUEH KH, et al., 2018). Os desfechos percentuais de redução da ansiedade nos voluntários do estudo, o qual eram portadores do distúrbio de sono, foram de $26,7 \%$, ao passo que o presente estudo obteve $19,03 \%$ em estudantes sem histórico de transtornos psiquiátricos ou comorbidades previamente relatadas, logo, acredita-se que a diferença percentual observada se dá mediante a presença do distúrbio do sono.

Realizando um comparativo com outras populações que não a dos estudantes universitários, também é possível averiguar evidências da eficácia da acupuntura auricular na diminuição da ansiedade. Um ensaio clínico randomizado com 180 enfermeiros, grupo usualmente submetido ao ambiente de estresse hospitalar, apresentou uma redução percentual da ansiedade em $13 \%$ no grupo que fez uso da auriculoterapia com sementes e 17\% naquele com aplicação da acupuntura auricular com agulhas (KUREBAYASHI LFS, et al., 2017). Os resultados foram obtidos ao se comparar o IDATE-E inicial e final, utilizando os mesmos questionários contemplando os escores de ansiedade preconizados no presente estudo. Observa-se ainda 
que, na população analisada, ocorreu diminuição ligeiramente maior dos níveis de ansiedade, quando comparada a redução de $13 \%$ observada no estudo citado, utilizando a auriculoacupuntura com sementes.

O uso da acupuntura auricular para a redução de ansiedade também pode ser observado em populações de dependentes químicos com acompanhamento longitudinal ao longo de meses, constatando uma redução de $29,54 \%$ ao longo das primeiras 5 semanas, com pouca alteração com o prolongamento do tratamento até o fim do terceiro mês (AHLBERG R, et al., 2016). Logo, a prática da acupuntura auricular pode ser relevante na melhora da qualidade de vida e de sintomas ansiosos em diversas populações, com ênfase nas relacionadas à área da saúde, realçando a importância dos resultados deste estudo.

Devido a seu efeito em potencial na redução da ansiedade, já foram realizadas pesquisas sobre a utilização da auriculoacupuntura para tratamentos de enfermidades como anorexia nervosa e procedimentos de amigdalectomia, sendo sua ação comparada ao uso de narcóticos e outros fármacos utilizados para redução da dor, agitação ou queixas somáticas (OLSSON A e LANDGREN K, 2020; SHAH AN, et al., 2019). Averígua-se, portanto, um potencial efeito de atenuar a agitação, observado com a utilização da terapia complementar em questão, que pode ser avaliado em estudos futuros.

Como levantado por alguns autores, a eficácia da acupuntura auricular não está restrita somente a redução da ansiedade, mas pode contribuir para a diminuição da tensão, agressividade e auxiliar no tratamento de dores crônicas (LORENT L, et al., 2016; MOURA CDC, et al., 2019a). Em revisão sistemática e metanálise desenvolvidas por Moura et al., constatou-se que, dos quinze estudos incluídos na revisão, doze (80\%) evidenciaram a efetividade da auriculoterapia para o controle das manifestações supracitadas (MOURA CDC, et al., 2019b). Por conseguinte, a presente pesquisa corrobora os achados desta revisão, trazendo dados que realçam a acupuntura auricular como método efetivo no combate de sintomas ansiosos.

A experiência, o conhecimento e a prática clínica do profissional da saúde que está executando o atendimento são fundamentais para o desfecho clínico, logo, a auriculoterapia conduzida por profissionais capacitados é mais efetiva (KUREBAYASHI LFS, et al., 2012).

\section{CONCLUSÃO}

O estudo evidenciou que, tanto os pacientes que receberam o protocolo placebo, quanto os pacientes que receberam o protocolo para ansiedade, tiveram seus níveis de ansiedade reduzidos. Entretanto, os participantes do grupo com intervenção para ansiedade apresentaram redução dos escores avaliados com significância estatística, o que não foi observado com os voluntários do grupo placebo. Os níveis médios de ansiedade examinados diminuíram até $19,03 \%$ nos pacientes do G1, comparados a 2,26\% do G2, ou seja, uma redução percentual oito vezes maior para o grupo com protocolo para ansiedade. Os resultados proporcionados pelo presente estudo são similares a publicações anteriores que trataram diretamente da relação entre o efeito da auriculoacupuntura e a redução da ansiedade. Sugerimos que sejam realizados novos estudos que avaliem a forma como a auriculoterapia atua nos sintomas de ansiedade e humor depressivo, utilizando amostras maiores, diferentes desenhos e durações.

\section{REFERÊNCIAS}

1. AHLBERG R, et al. Auricular acupuncture for substance use: A randomized controlled trial of effects on anxiety, sleep, drug use and use of addiction treatment services. Substance Abuse: Treatment, Prevention, and Policy, 2016; 11(1): $1-10$.

2. AMORIM D, et al. Acupuncture and electroacupuncture for anxiety disorders: A systematic review of the clinical research. Complementary Therapies in Clinical Practice, 2018; 31: 31-37.

3. BRASIL. Ministério da Saúde. Ansiedade. Biblioteca Virtual em Saúde. 2011. Disponível em: https://bvsms.saude.gov.br/dicas-em-saude/470-ansiedade. Acesso em: 07 dez. 2020.

4. BRASIL. Ministério da Saúde. Secretaria de Atenção à Saúde. Departamento de Atenção Básica. Política nacional de práticas integrativas e complementares no SUS: atitude de ampliação de acesso /Ministério da Saúde. Ministério da Saúde, 2015. Disponível em: http://bvsms.saude.gov.br/bvs/publicacoes/politica_nacional_praticas_integrativas_complementares_2ed.pdf 
5. BUCHANAN TM, et al. Reducing Anxiety and Improving Engagement in Health Care Providers Through an Auricular Acupuncture Intervention. Dimensions of Critical Care Nursing, 2018; 37(2): 87-96.

6. CHUEH KH, et al. Effects of Auricular Acupressure on Sleep Quality, Anxiety, and Depressed Mood in RN-BSN Students with Sleep Disturbance. Journal of Nursing Research, 2018; 26(1): 10-17.

7. CROCQ MA. A history of anxiety: from Hippocrates to DSM. Dialogues in Clinical Neuroscience, 2015; 17(3): 319325.

8. ERRINGTON-EVANS N. Acupuncture for Anxiety. CNS Neuroscience \& Therapeutics, 2012; 18(4): $277-284$.

9. GODOY LD, et al. A Comprehensive Overview on Stress Neurobiology: Basic Concepts and Clinical Implications. Frontiers in Behavioral Neuroscience, 2018; 12(127): 1-23.

10. GRANER KM, CERQUEIRA ATDAR. Integrative review: Psychological distress among university students and correlated factors. Ciencia e Saude Coletiva, 2019; 24(4): 1327-1346.

11. HALLER $\mathrm{H}$, et al. Complementary therapies for clinical depression: An overview of systematic reviews. BMJ Open, 2019; 9(8): 1-15.

12. HASTY LM, et al. When anxiety becomes my propeller: Mental toughness moderates the relation between academic anxiety and academic avoidance. Br J Educ Psychol, 2020: 1-23.

13. KEYES CLM, et al. The relationship of level of positive mental health with current mental disorders in predicting suicidal behavior and academic impairment in college students. Journal of American College Health, 2012; 60(2): $126-133$.

14. KLAUSENITZ C, et al. Auricular acupuncture for exam anxiety in medical students - A randomized crossover investigation. PLoS ONE, 2016; 11(12): 1-9.

15. KUREBAYASHI LFS, et al. Eficácia da auriculoterapia para estresse Segundo experiencia do terapeuta: ensaio clínico. ACTA Paulista de Enfermagem, 2012; 25(5): 694-700.

16. KUREBAYASHI LFS, et al. Auriculotherapy to reduce anxiety and pain in nursing professionals: a randomized clinical trial. Revista Latino-Americana de Enfermagem, 2017; 25(0).

17. LAN Y, et al. Auricular acupuncture with seed or pellet attachments for primary insomnia: A systematic review and meta-analysis. BMC Complementary and Alternative Medicine, 2015; 15(1).

18. LORENT L, et al. Auricular Acupuncture Versus Progressive Muscle Relaxation in Patients with Anxiety Disorders or Major Depressive Disorder: A Prospective Parallel Group Clinical Trial. JAMS Journal of Acupuncture and Meridian Studies, 2016; 9(4): 191-199.

19. MOURA CDC, et al. Acupuntura auricular para dor crônica nas costas em adultos: revisão sistemática e metanálise. Revista da Escola de Enfermagem da USP, 2019a; 53: e03461.

20. MOURA CDC, et al. Auriculoterapia efeito sobre a ansiedade TT - Efecto de la auriculoterapia sobre la ansiedad TT - Effect of auriculotherapy on anxiety. Rev Cubana Enferm, 2019b; 7(6): 0.

21. OLSHAN-PERLMUTTER M, et al. Auricular acupressure reduces anxiety and burnout in behavioral healthcare. Applied Nursing Research, 2019; 49: 57-63.

22. OLSSON A, LANDGREN K. Getting Well Is More Than Gaining Weight - Patients' Experiences of a Treatment Program for Anorexia Nervosa Including Ear Acupuncture. Issues in Mental Health Nursing, 2020; 41(4): 328-338.

23. PEREIRA C, et al. Depression's Unholy Trinity: Dysregulated Stress, Immunity, and the Microbiome. Ann. Rev. Psychol., 2019; 71(21): 21.1-21.30.

24. QUEK TTC, et al. The global prevalence of anxiety among medical students: A meta-analysis. International Journal of Environmental Research and Public Health, 2019; 16(15).

25. RABISCHONG P, TERRAL C. Scientific basis of auriculotherapy: State of the Art. Medical Acupuncture, 2014; 26(2): 84-96.

26. ROTENSTEIN LS, et al. Prevalence of depression, depressive symptoms, and suicidal ideation among medical students a systematic review and meta-analysis. JAMA - Journal of the American Medical Association, 2016; 316(21): 2214-2236.

27. SHAH AN, et al. Auricular acupuncture for adult tonsillectomy. The Laryngoscope, 2019; 130(8): 1907-1912.

28. SMITH CA, et al. Acupuncture for depression. Cochrane Database of Systematic Reviews, 2018; 2018(3): 14651858.

29. VICKERS AJ, et al. Acupuncture for chronic pain: individual patient data meta- analysis. Arch Intern Med, 2012; 172(19): 1444-1453.

30. VIEIRA A, et al. Does auriculotherapy have therapeutic effectiveness? An overview of systematic reviews. Complementary Therapies in Clinical Practice, 2018; 33(August): 61-70.

31. WIRZ-RIDOLFI A. The History of Ear Acupuncture and Ear Cartography: Why Precise Mapping of Auricular Points Is Important. Medical Acupuncture, 2019; 31(3): 145-156.

32. WORLD HEALTH ORGANIZATION. 2017. Depression and other common mental disorders: global health estimates. World Health Organization. Disponível em: https://apps.who.int/iris/bitstream/handle/10665/254610/WHO-MSD-MER2017.2-eng.pdf. Acesso em: 07 dez. 2020.

33. WU C, et al. Transcutaneous auricular vagus nerve stimulation in treating major depressive disorder A systematic review and meta-analysis. Medicine (United States), 2018; 97(52).

34. YARIBEYGI H, et al. The impact of stress on body function: A review. EXCLI Journal, 2017; 16: 1057-1072. 